\title{
Pulmonary Nodular Amyloidosis in a Patient with Sjogren Syndrome
}

\author{
Yeonmi Ju, Won-Seok Lee, Wan-Hee Yoo
}

Division of Rheumatology, Department of Internal Medicine, Chonbuk National University Medical School and Research Institute of Clinical Medicine, Jeonju, Korea

\section{Image in Rheumatology}

A 61-year-old woman was admitted for assessment of multiple nodules incidentally detected on chest radiography (Figure 1A).
Six months previously, we diagnosed Sjögren's syndrome upon observation of symptoms of dryness of the mouth and eyes, positive Schirmer's test, positive fluorescent antinuclear anti-
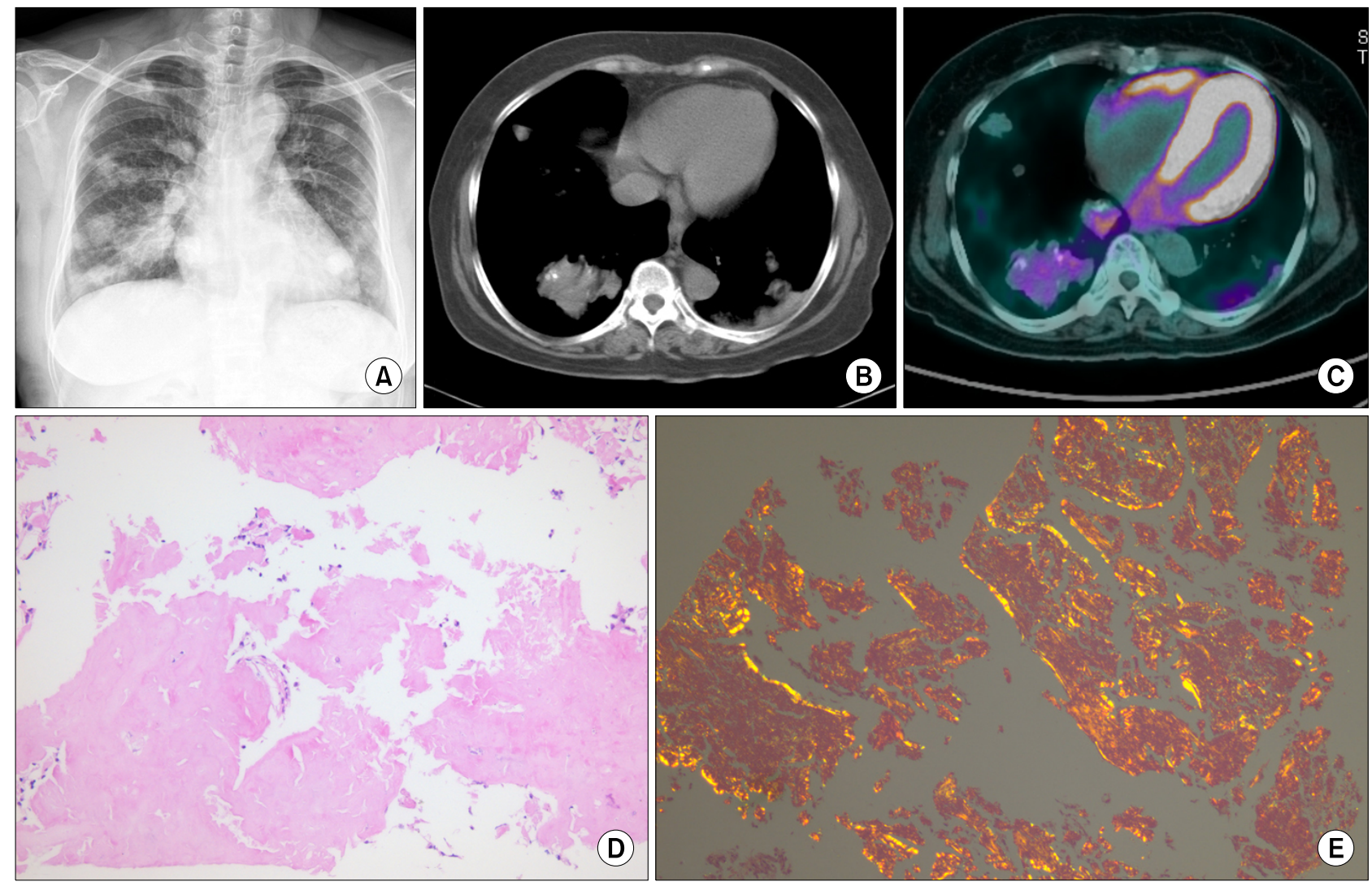

Figure 1. Multiple nodules and a mass in both lung field. Histology showing dense accumulation of typical amorphous eosinophilic material and calcification, and Congo red stain showed apple-green birefringence.

$<$ Received: April 30, 2014, Accepted : June 24, 2014>

Corresponding to : Wan-Hee Yoo, Division of Rheumatology, Department of Internal Medicine, Chonbuk National University Medical School and Research Institute of Clinical Medicine, San 2-20 Geumam-dong, Deokjin-gu, Jeonju 561-180, Korea. E-mail : ywhim@jbnu.ac.kr

pISSN: 2093-940X, eISSN: 2233-47I8

Copyright (C) 2014 by The Korean College of Rheumatology

This is a Free Access article, which permits unrestricted non-commerical use, distribution, and reproduction in any medium, provided the original work is properly cited 
body test, elevated levels of anti-Ro antibodies, and positive salivary gland scintigraphy findings. Spirometry showed moderate restrictive ventilatory defect (FVC: $1.9 \mathrm{~L}, 65 \%$ of predicted, DLco: 70\%). Chest tomography revealed a mass measuring $4.7 \times 7.8 \mathrm{~cm}$ on the right lower lobe, multiple calcified nodules in both lungs (Figure 1B). Fluorodeoxyglucose positron emission tomography showed multiple hypermetabolic amorphous masses (Figure 1C). Lung tissue specimens showed dense accumulation of typical amorphous eosinophilic material and calcification (Figure 1D, hematoxylin-eosin stain, 40×), and Congo red staining showed apple-green birefringence (Figure 1E). Immunohistochemistry using specific antibodies to the $\lambda$ light chain confirmed the diagnosis of light-chain (AL) $(\lambda)$ amyloidosis. Amyloidosis rarely causes diffuse septal and nodular pulmonary infiltrates in SS $(1,2)$. A review of literature showed that lymphoma is associated in $33 \%$ of cases (2). Most patients require a lung biopsy to diagnose pulmonary amyloi- dosis and exclude associated lymphoma. The treatment of diffuse nodular amyloidosis in SS is complicated by its rarity (2). We suggested treatment with high-dose steroids followed by oral or parenteral cyclophosphamide, but the patient did not agree. We performed computed tomography (CT) every 6 months for 1 year; during this period, her condition remained unchanged, without any significant deterioration or evidence of systemic amyloidosis or lymphoma. Clinicians should be aware that nodular or cystic lung lesions in primary SS represent amyloidosis, and a lung biopsy must be performed for confirmation.

\section{References}

1. Strimlan CV. Pulmonary involvement in Sjögren's syndrome. Chest 1986;89:901.

2. Rajagopala S, Singh N, Gupta K, Gupta D. Pulmonary amyloidosis in Sjogren's syndrome: a case report and systematic review of the literature. Respirology 2010;15: 860-6. 